$\Rightarrow$ PAIN

\section{Long-term outcomes of TRMDSC for chronic orchialgia}

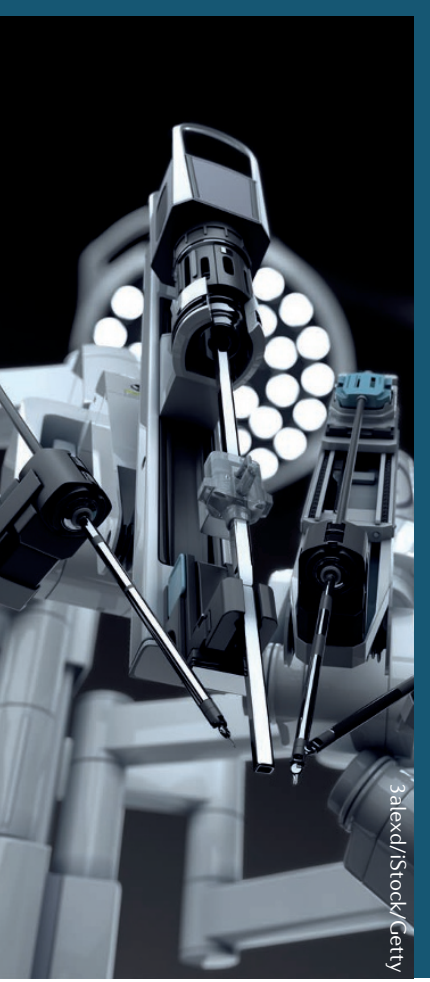

Targeted robotic-assisted microsurgical denervation of the spermatic cord (TRMDSC) could have long-lasting beneficial effects for patients with refractory chronic orchialgia, according

Conservative treatments for chronic orchialgia are often ineffective and surgical options are not always successful. Standard MDSC was introduced as an option for treatment in the 1970s, but it involves skeletonization of the entire spermatic cord and can lead to adverse effects such as lymphocele and haematoma formation. TRMDSC was developed as a less aggressive option that targets specific nerve fibres and the new study reports on long-term outcomes of this procedure.

\section{Calixte et al. performed a} retrospective review of 872 TRMDSC procedures performed in 772 patients (median age 41 years) between October 2007 and July 2016. Included patients had experienced chronic testicular pain to a recent study. for at least 3 months (median 6 years), had failed standard treatments, and had demonstrated temporary but complete resolution of pain with local anaesthetic block of the spermatic cord.

Pain was assessed using the visual analogue scale and a standardized validated pain score (the pain impact questionnaire PIQ-6) before surgery, and 1 month, 3 months, 6 months, 1 year, 2 years, 3 years, and 4 years after surgery. Follow-up data were available for 860 TRMDSC procedures and median follow-up time was 2 years.

426 TRMDSC procedures ( $49 \%$ ) were associated with complete resolution of pain, 292 procedures (34\%) were associated with a $50 \%$ reduction in pain and 142 procedures $(17 \%)$ were associated with continued pain. PIQ-6 data showed that $67 \%$ of procedures were associated with a notable reduction in pain at 6 months, $68 \%$ at 1 year, $77 \%$ at 2 years, $86 \%$ at 3 years, and $83 \%$ at 4 years. Complications included haematoma in 23 patients
(2.67\%), wound infection in 13 patients (1.5\%), wound dehiscence in nine patients $(1.04 \%)$, and seroma in four patients $(0.47 \%)$.

In order to assess whether sperm parameters were affected by the TRMDSC procedure, the researchers performed a subset analysis on 319 patients who underwent TRMDSC and varicocelectomy to treat painful varicocele. Overall, $73 \%$ of these patients had a substantial reduction in pain, $65 \%$ showed improvements in sperm count and motility, and $66 \%$ demonstrated increases in serum testosterone levels.

Testicular loss occurred in one patient after TRMDSC. This patient had previously experienced severe pelvic injuries and undergone a number of pelvic and groin exploratory procedures during which testicular and vasal arteries had been damaged. The researchers say that they now suggest alternative treatments to such patients.

Rebecca Kelsey

ORIGINAL ARTICLE Calixte, N. et al. Targeted robotic assisted microsurgical denervation of the spermatic cord for the treatment of chronic orchialgia or groin pain: single center, large series review. J. Urol. http://dx.doi.org/10.1016/ j.juro.2017.10.030(2017) 